\title{
Control of Nao Robot Arm using Myo Armband
}

\author{
Ramkumar A, Akhil Krishna U, Madhan M S, Prajit K K
}

\begin{abstract}
Life becomes less complex, resourceful and very educative thanks to the use of smart devices like the Myo armband and Nao robots. This work discusses about the use of Myo Armband which is a wireless device for interacting with other devices such as computer, robots. It uses myographic sensor signals to control the robot. This involves developing a digital control interface to control the robot with the help of Myo Gesture Control Armband System. A PC loaded with Ubuntu OS acts as a control unit. It interfaces the inputs of the Myo band to control the Nao robot, thereby we can control the movement of the Nao robot by using its interactions. In this case we use Nao, an educational robot. PyoConnect is a Linux alternative to MyoConnect a scripting software for programming the Myo band in Windows. The Pyoconnect software is used to connect the Myo with the Ubuntu operating system. NAOqi is a programming framework used to program the NAO. By importing the NAOqi module in the python script we can access the different functions of the Nao robot. Nao robot which supports network communication protocols are Ethernet and Wi-Fi. The interface between Nao and Myo is achieved through IP (Internet Protocol). Based on the hand gestures recorded by Myo armband, the Nao Robot Arm's can be controlled.
\end{abstract}

Keywords -- NAO, MYO, NAOqi, Pyoconnect, Myoconnect, Linux, PythonScript, Ethernet Protocol

\section{INTRODUCTION}

Myo is a wearable device where based on preset gestures it creates a controlling platform to other electronic systems. Electromyography is an technique which is used for evaluating and recording the electrical activity which has been produced by skeletal muscles [1].These Electromyography which records the electric potential is produced by the activity in a muscle cells during its contraction, and it can be detected through electrodes applied on skin. The use of these signals is very feasible for analysis of movements and controlling of some electronic devices, since for each movement it has different modes of muscle activation, which are reflected in different signatures or patterns for device control. In this paper we describe the method of using the myo to control the nao humanoid robot. The communication between ubuntu and nao robot can be made through IP(Internet Protocol) . PyoConnect which is an software used for communication between $\mathrm{pc}$ and myo armband. Using the Naoqi module in the python script we can control the Nao's arm

Revised Manuscript Received on July 18, 2019.

Ramkumar A, Department of Mechatronics Engineering, Kumaraguru College of Technology, Coimbatore, Tamil Nadu, India.(E-mail:. ramkumar.a.mce@kct.ac.in)

Akhil Krishna U, Department of Mechatronics Engineering, Kumaraguru College of Technology, Coimbatore, Tamil Nadu, India.(Email:. akhil.14mc@kct.ac.in)

Madhan M S, Department of Mechatronics Engineering, Kumaraguru College of Technology, Coimbatore, Tamil Nadu, India.(E-mail:. madhan2.14mc@kct.ac.in)

Prajit K K, Department of Mechatronics Engineering, Kumaraguru College of Technology, Coimbatore, Tamil Nadu, India.(E-mail:. prajit.14mc@kct.ac.in)

\section{BACKGROUND OF INVENTION}

\section{A Introduction}

The wearable device, Myo armband create a controlling platform to other electronic devices with preset gestures synced to its program to acquire inputs form the myographic impulses of the arm. Electromyography is used for recording and evaluating the electrical activity which has been induced by skeletal muscles [1]. During the contraction of muscles the electric potential will be generated and it can be recorded by electromyography. For each muscle movements there will be different patterns or signature will be reflected so it can be very feasible to control the electronic devices and to analyze the movements. In our project we are using these myo to control the nao robot. The communication between raspberry pi and nao robot can be made through TCP/IP Protocol. PyoConnect which is an software used for communication between raspberry pi [14] and myo armband. Using the Naoqi module in the python script we can control the Nao's arm.

\section{$B$ Interactive humanoid Robots}

The NAO is an interactive robot which with its monitor program can be used to verify the internal memory of the robot and help with its functions.[6] Choreographe is a graphical tool for programming the Nao robot. It uses diagrams to help Nao what to perform. Its movements are given by timeline features function. Nao's movements are also given using box libraries and FTP. Choreographe uses python script boxes. NAOqi allows to use Linux, $\mathrm{C}++$ and cross compile platform. Python [13] can be and for implementing inverse Kinematics and forward kinematics calculations.[6]

\section{Nao Kinematics}

It provides NAO's joint structure information for each joint. The Denavit-Hartenberg (DH) method is used to explain the calculation for forward kinematics. In addition, Python will be used to create an actual forward kinematics calculation program. We also come to know about the use of Python and calculations for inverse kinematics to implement the calculation program of inverse kinematics for NAO's arm. [6]

\section{Application of Myo in Robot Interface}

It discusses the myoelectric signals which have been used in the various applications to control some of the electronic devices which aiming the development of a digital controlling interface with Myo Armband System. The movement of a robot can be controlled by this interface and

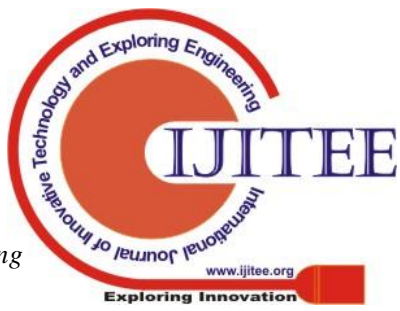


its interaction with the surroundings, in this case the robot which has been mentioned here is PeopleBot, which is specifically designed for the purpose of home necessities. It allowing an assessment during the operation of controlling devices with Inertial Measurement Unit and myoelectric signals.[10]

\section{E Simultaneous human feedback and Control}

In simultaneous feedback signals and human control there are various drawbacks and benefits of incorporating in the training of a robotic agent. While robotic human-machine interfaces has resulted in an increasing gap between user control approaches and the number of robotic actuators which can be controlled. One way to address this gap is to transfer the power to the robot. Semi-autonomous actions of the robotic agent can then be shaped and refined by human feedback, simplifying user control. Training with feedback was used in previous cases or they have used indirect control signals. This paper tells how a human can provide concurrent feedback signals and real-time myoelectric control signals to train a robot's actorcritic reinforcement learning control system. On a simple movement task, by using a simulated and physical robotic system shows training performance when reward is derived from the human, from the environment, and from the two combinations. The results indicated including human generated feedback in learning algorithms for the humanmachine interactive domain. [11]

\section{F Robotic arm manipulation control using myo}

The control of two manipulating arms using EMG signals captured with a gesture control and motion control device is performed using Myo Armband. To control the degrees of freedom of two different manipulating arms where it uses the electromyographic signal recognition to achieve the work. Initially the control signals were acquired by analyzing the Myo armband and arms of the robot were characterized for associate its freedom degrees to the moves of an electromyographic signal. Finally, a test scenario was implemented to validate the robotic arms control through the moves of the user arms using the Myo's. [5]

\section{G Interfacing of Myo Armband System to Control a Robot}

This paper discusses the application of myoelectric signals to control electronic devices aiming the development of a digital controlling interface with Myo Gesture Control Armband System. Through this interface it is possible to control the movement of a robot and its interaction with the environment, in this case the robot being PeopleBot, a robot designed for home necessities. Thus, allowing an assessment during the operation of controlling devices with Inertial Measurement Unit (IMU) and myoelectric signals, benefits and drawbacks of working with this technology which has been discussed. [4]

\section{$H$ Visual control of robotic arm}

Myo device detects electrical activities from different parts of the forearm muscles by using electromyography (EMG) sensors. It uses gyroscope and an accelerometer. EMG sensors detect and provide important muscles data reading compared with other types of sensors. The datas from gyroscope, accelerometer and EMG sensors of the myo armband send to a computer via Bluetooth and transmitted data is used to control a arm virtual robot which was built in Unity 3D. Arms of the virtual robot based on accelerometer, gyroscope, and EMG sensors have different features electromyographic reading is used to control by using the relaxation of muscles and parameters of tension. Consequently, the arm of a virtual robot based on electromyographic is mostly preferred for a hand amputee to arm of the virtual robot based on an accelerometer and a gyroscope. [2]

\section{Robotic system control using myo}

The recent trends in robots are discussed for the Human gesture interaction with robotic system in control system for its more precise, efficient and sustainability control. Design approach of a robotic system by using muscle sensor is discussed here. The muscle sensor used is a Myo armband sensor which works based on the contraction and relaxation of muscle, performed using human gesture. This Paper aims to design the structure of robotic arm by using the servo motor with five degrees of freedom to be controlled by the Myo[2]. They use Euler mechanics in to find the arm position. It is carried out using gesture control and the data can be sent to microcontroller to control the robotic arm. A human can control the Robot with his arm. Besides, the communication constructed between the signal of Myo armband and the robotic system. The human interactive implementation can be used to modified and control various robotic system. [1]

\section{$J M Y O A R M B A N D$}

The Myo Armband is a device which is used in controlling the several electronic devices and it can also be used by the humans to interacting with the robots. This Myo armband has an eight-stainless steel electromyographic sensors. The signals received from the EMG sensor which will behave similar to other surface electrodes. From the muscle activities the electric potential will be generated. This electric potential will be very small. The range of a potential will be sub millivolt and this signal can be very sensitive. Varied source of electrical noise such as wallelectricity will induce a noise and it can interfere with the myo readings. This Myo armband has a nine-axis inertial measurement unit. It contains a three-axis gyroscope, three axis accelerometers and a three-axis magnetometer. From these units, where the wearer's arm orientation and movement can be determined by analysing with the spatial data provided. The positioning of the armband in terms of roll, pitch and yaw will be indicated by these orientation data. The angular velocity of the armband will be provided in the vector format and the accelerometer will represents the acceleration where the myo armband is undergoing at a given time. However, the Myo armband which is mainly suited for to determine the relative positioning of the arm rather than the absolute position considering and applying suitable pattern recognition algorithm. The Myo armband which has been designed to work in the forearm, that is mainly in the upper forearm. For a more constrained grip

Published By: 
where the sizing clips is available and it is well suited for smaller arms. This electromyographic sensors is unlike the other, where the Myo wearer does not requires to shave the area around where the armband will be worn. Then setup procedures in experimental or real-world environments will be lot easier.

\section{K NAO ROBOT}

Nao is an autonomous humanoid robot which is developed by the Aldebaran Robotics. This Nao robot can be programmable and it is mainly used for the educational purposes. This Nao robot which has 25 degrees of freedom. Those tension which can be individually controlled through an actuator and an electric motor. The Ethernet or wireless networking can be used by the user to program and control the robot. This Nao robot which mainly features an inertial measurement unit with accelerometer, gyrometer and four ultrasonic sensors which will provide a Nao with the stability and positioning within space. The OS which powers the robot's multimedia system, it includes two HD cameras (computer vision, including facial and shape recognition), four microphones (voice recognition and sound localization) and two speakers (multilingual text-to-speech synthesis). The software suite which comes with Nao robot that includes Choregraphe which is graphical programming tool. This Nao robot which can be controlled by a specialized Linux-based operating system, by using these NAOqi. NAOqi is the programming framework which can be used to control all the hardware components of the NAO robot. The software's such as the Gostai URBI Studio, Cyberbotics Webots, and Microsoft Robotics Studio where this software furthermore compatible with Nao.

\section{NAO AND MYO INTERFACE \& RESULTS}

\section{A Working of Myo Armband}

MYO Armband which contains eight electromyographic sensor in order to detect muscle movements around your forearm and hand gestures. This Armband also contains a magnetometer, an accelerometer and a gyroscope to track arm movements, such as moving or spinning. Myo will sends the information to the user by vibrating when it detects the gesture or when PC is synced with it. A micro USB port is used for recharging the battery, where it can be used for full day. When a gesture is made it will starts to detect the muscle movements. To guess the corresponding gestures, it has to be trained and it will respond accordingly to the performed gesture. The following gestures which are currently supports by the myo:

- Double-tap: It is normally used to unlock Myo, so it starts to detect other gestures. Tap your middle finger with your thumb twice.

- Fist: The user must close the hand in a fist to use this gesture.

- Spread Fingers: To use this gesture the user must spread all five fingers.

Wave in: To use this gesture the user must wave his hand towards his body. Wave out: To use this gesture the user must wave his hand contrary to the body.

\section{$B$ Working of NAO}

The overall operation of a Nao humanoid robot can be managed by the Naoqi framework. It enables the communication system and the user interactions. By using the Choregraphe software we can connect the real robot by using the port and the IP address. By using the NAOqi embedded software we can connect to the nao robot by programming it using various languages like python, $\mathrm{c}++, \mathrm{c}$. This Naoqi will act as user and communication for the Nao robot system. To program the Nao robot the Naoqi programming framework is used. The synchronization, parallelization, event and resource management are the main features of Naoqi. The communication will be established between the modules like video, motion and audio by this Naoqi. This NAOqi which also enables the programming and information sharing through ALMemory (robot memory) and communication between modules like motion, video and audio which also serve the other roles. The communication between the actuator and sensors excluding the cameras and sound is managed by the Device Communication Manager. This communication manager is a part of this NAOqi system. It is a one of the software module of the Nao robot. NAOqi framework which works by using the Audio module, Choregraphe, Monitor, and motion module where the information will be passed to each other. By the commands and Broker deliver information where the NAOqi is executed.

\section{Communication protocol}

There are two methods which is available for establishing a connection between the NAO and personal computer. First method is to plug a personal computer with an Ethernet cable and the Nao is also to be connected with the same Ethernet cable.

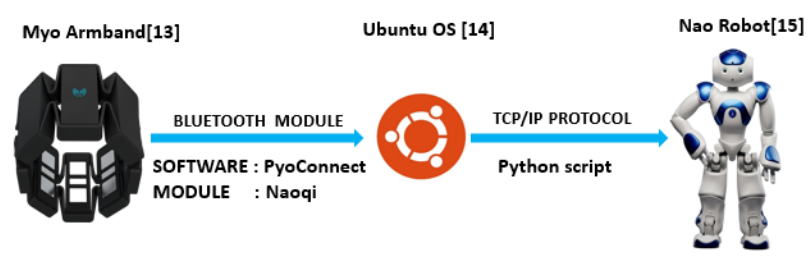

Figure 1 Communication Protocol

The other method is by using the WIFI. The Nao humanoid robot and personal computer have to be connected with the same WIFI connection. There are two variants, the fixed IP address which can be provided by the Ethernet cable connection. For the WIFI connection, the dynamic IP address will be provided 


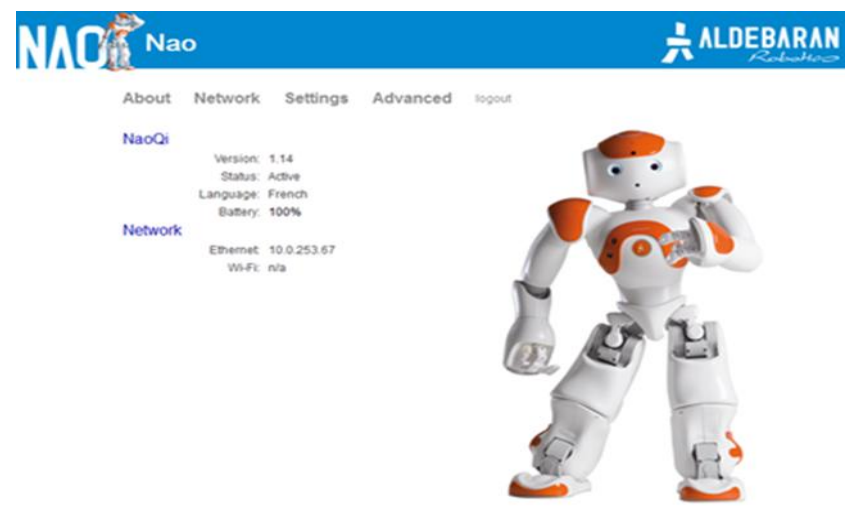

Figure 2 Nao Communication

We used the fixed IP address that is ethernet cable is used to make a communication with the Nao and Ubuntu operating system.

\section{Interfacing Nao with Myo}

To interface Myo and Nao we should connect Myo Armband with the personal computer initially by using this Pyoconnect in the ubuntu operating system. This PyoConnect is a Linux based alternative to MyoConnect scripting that works in Windows operating system. The PyoConnect uses the Python language/ PyScript to interface myo with the Ubuntu operating system. The Nao humanoid robot and Ubuntu operating system should be connected to same Ethernet connection/ server. We have used the Internet Protocol to connect the Nao with the Ubuntu operating system.

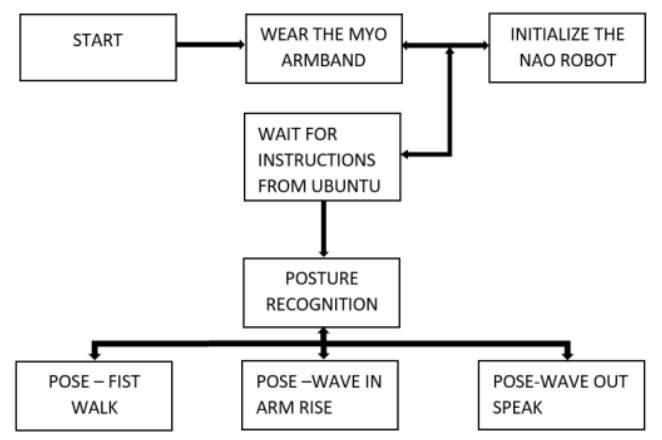

Figure 3 Algorithm to interface Nao with Myo

The NAO operations are controlled by the Naoqi programming framework. It controls and establishes the communication between modules such as motion, audio and video by using this Naoqi framework. To control the Nao arm we have to use the Naoqi in the Ubuntu OS by importing the NAOqi module in the python script is performe.

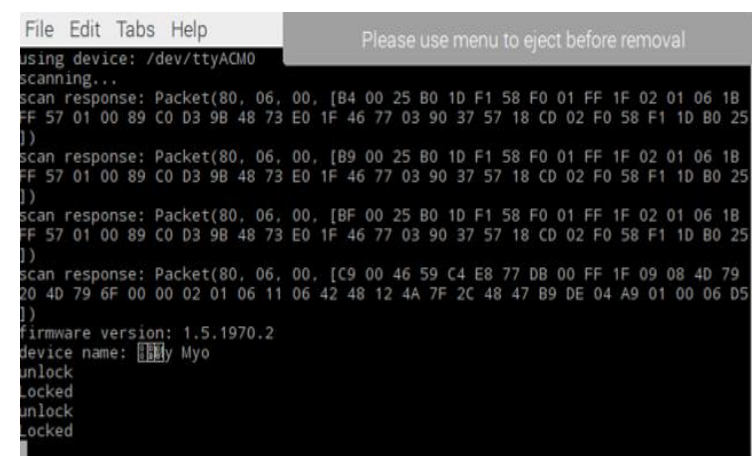

Figure 4 Programing in Ubuntu for MYO

We can access the different motion modules of the NAO using this NAOqi framework. We have to import ALProxy from these Naoqi for to control the motions of Nao. It is a client server protocol that is used for communication inside the Nao's software. The creation of an ALProxy module is to be performed. This process is called as the method. A call for the function block is created for accessing the method.

\section{E Results and Discussions}

Nao Robots operations are controlled by the inputs form the myo armband which is compiled in the ubuntu OS platform. The functional interface to the control the nao robot using the myoelectric technology of the myo armband has been implemented. Nao robot was programmed for various myo inputs and it was programmed to perform movements and grips. Myo input controlling response are very fast since it uses the gyroscope, accelerometer and control to its raw data. Though there are only few gesture that are defined to the user arm positions and movements, various other functions can create combinations of one other, this overcomes some of this limitation of less gesture movements and is very practical/comfortable for user to control the Nao robot.

The following functions were performed by using the Myo armband to connect to the Nao robot.

- Double-tap: It is normally used to unlock Myo, so it starts to detect other gestures. Tap your middle finger with your thumb twice.

- Fist: The user must close the hand in a fist to use this gesture.

- Spread Fingers: To use this gesture the user must spread all five fingers.

- Wave in: To use this gesture the user must wave his hand towards his body. Wave out: To use this gesture the user must wave his hand contrary to the body.

The fingers spread posture is set as the input in the program, if the gesture is triggered the program loop is turned $\mathrm{ON}$ or the state is set true. This command enables the robot to move forward in a straight path until held upon that posture. To read the other gestures we have to tap our middle finger with our thumb twice. When the fist gesture is read by Myo Armband, it triggers the corresponding program loop. This fist gesture which enables the Nao robot to close his arm. When the wave in gesture is recognized by Myo Armband, as the input to the program it will enables

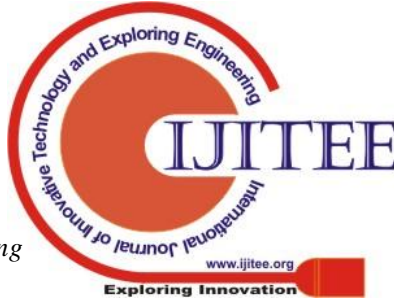


the Nao humanoid robot to speak what has been coded in the program. The Wave out posture is set as the input in the program, if the gesture is triggered the program loop is turned ON. This command enables the robot to rest state.

There were various errors encountered during the process of connecting the Nao robot with the Myo band. A few of the errors were,

- Import error

- Version mismatch

The version of Python does not match the one with which the Python SDK was made.

\section{CONCLUSIONS}

The Nao robot, a humanoid robot has its capabilities of replicating a few tasks of a human. It got sensors and motors for motion, vision and voice. Using the Myo armband we were able to link the gestures of the Myo to perform a specific action in the Nao robot. The gestures such as fingers spread out, fist, wave-in and wave-out are used as an input for the Nao. The gestures trigger a specific movement or a control in the robot. The NAOqi framework in the robot supports the use of Pyscript for programming the Nao. Similarly, using PyoConnect we get the myographic signals in a python script page from the Myo band gestures. By linking the both gestures and the NAOqi framework the control of the Nao robot is performed.

\section{REFERENCES}

1. A. Doswald, "Using biosignals to control the Nao robot by," no. January, 2013.

2. A. Ganiev and K. Lee, "Study on Virtual Control of a Robotic Arm via a Myo Armband for the Self- Manipulation of a Hand Amputee," vol. 11, no. 2, pp. 775-782, 2016.

3. C. Lagrand, M. Van Der Meer, and A. Visser, "Ros Nao Tutorial Simulation \& Real robots," 2016.

4. G. D. Morais, L. C. Neves, A. A. Masiero, and M. C. F. Castro, "Application of Myo Armband System to Control a Robot Interface," vol. 4, no. Biostec, pp. 227-231, 2016.

5. P. U. Murillo, "Individual Robotic Arms Manipulator Control Employing Electromyographic Signals Acquired by Myo Armbands," vol. 11, no. 23, pp. 11241-11249, 2016.

6. P. Sarjana and M. Ii, "ROBOTIC SYSTEM DESIGN CONTROL BY USING ARMBAND SENSOR “.This Report Is Submitted In Fulfillment Of Requirement For The Bachelor Degree of Electronic Engineering (Computer Engineering) Faculty of Electronic And Computer Engineering University Technical Malaysia Malacca June 2015 ii,” no. June, 2015.

7. "An SSVEP based BCI to control a humanoid robot by using portable EEG device."published in Engineering in Medicine and Biology Society,2013.

8. "Using Myo to control Robots: 5 Examples" by Gadget Junkie - April 16.

9. A. Robotics, "Using nao : Introdution to interactive humanoid robots." 20

10. Application of Myo Armband System to Control a Robot InterfaceGabriel Doretto Morais, Leonardo C. Neves, Andrey A. Masiero and Maria Claudia F. Castro Centro Universit’ario da FEI, Av. Humberto Alencar Castelo Branco 3972, S ao Bernardo do Campo, Brazil

11. Simultaneous Control and Human Feedback in the Training of a Robotic Agent with Actor-Critic Reinforcement Learning Kory Mathewson and Patrick M. Pilarski ,Departments of Computing Science and Medicine,University of Alberta, Edmonton, Alberta, Canada,[korym, pilarski] @ ualberta.ca

12. Controlling a Robot Using a Wearable Device (MYO), 1Mithileysh Sathiyanarayanan, Tobias Mulling, Bushra
Nazir,School of Computing, Engineering and Mathematics,University of Brighton, United Kingdom

13. Premalatha J, Shanthi Vengadeshwari R, Veerendha A, "Optimization of transmission tower using Genetic Algorithm", International Journal of Civil Engineering and Technology Volume 8, Issue 9, September 2017, pp. 654662.

14. Shivappriya $\mathrm{S}$ N, Dhivyapraba R, Kalaiselvi A, Alagumeenakshi M, "Telemedicine Approach for Patient Monitoring System using IOT", Research Journal of Engineering and Technology, 8(3): July-September 